\title{
FLORESCIMENTO E FRUTIFICAÇÃO DE MANGUEIRA COM USO DE PACLOBUTRAZOL, ETHEPHON E NITRATO DE CÁLCIO ${ }^{1}$
}

\author{
VANDER MENDONÇA ${ }^{2}$, SEBATIÃO ELVIRO DE A. NETO ${ }^{3}$, OSCAR MARIANO HAFLE $^{3}$, \\ JOSIVAN BARBOSA MENEZES ${ }^{4}$ E JOSE DARLAN RAMOS ${ }^{5}$
}

\begin{abstract}
RESUMO - Este trabalho objetivou testar diferentes doses de Paclobutrazol (PBZ), ethephon e nitrato de cálcio na indução do florescimento e na produção da mangueira (Mangifera indica L.) cv. Tommy Atkins, localizada no pomar didático da ESAM em Mossoró-RN, no ano de 1999/2000. O delineamento experimental utilizado foi em blocos casualizados, no esquema fatorial 2x2x3, assim distribuídos: $\mathrm{T}_{1} 1000 \mathrm{mg} . \mathrm{L}^{-1}$ de PBZ+ 2\% de nitrato de cálcio; $\mathrm{T}_{2} 1000 \mathrm{mg} \cdot \mathrm{L}^{-1} \mathrm{de} \mathrm{PBZ}+2 \%$ de nitrato de cálcio $+1,0 \mathrm{~mL} . \mathrm{L}^{-1}$ de ethephon; $\mathrm{T}_{3} 1000 \mathrm{mg} . \mathrm{L}^{-1}$ de PBZ + 2\% de nitrato de cálcio $+3,0 \mathrm{~mL} \cdot \mathrm{L}^{-1}$ de ethephon; $\mathrm{T}_{4} 1000 \mathrm{mg}$. $\mathrm{L}^{-1}$ de PBZ + 3\% de nitrato de cálcio; $\mathrm{T}_{5} 1000 \mathrm{mg} . \mathrm{L}^{-}$ 1 de PBZ + 3\% de nitrato de cálcio + 1,0 mL. $\mathrm{L}^{-1}$ de ethephon; $\mathrm{T}_{6} 1000 \mathrm{mg} \cdot \mathrm{L}^{-1}$ de PBZ + 3\% de nitrato de cálcio + 3,0 mL. $\mathrm{L}^{-1}$ de ethephon $; \mathrm{T}_{7} 1500 \mathrm{mg} . \mathrm{L}^{-1}$ de $\mathrm{PBZ}+2 \%$ de nitrato de cálcio; $\mathrm{T}_{8} 1500 \mathrm{mg} . \mathrm{L}^{-1} \mathrm{de} \mathrm{PBZ}+2 \%$ de nitrato de cálcio $+1,0 \mathrm{~mL} . \mathrm{L}^{-1}$ de ethephon; $\mathrm{T}_{9} 1500 \mathrm{mg} . \mathrm{L}^{-}$ ${ }^{1}$ de $\mathrm{PBZ}+2 \%$ de nitrato de cálcio $+3,0 \mathrm{~mL} \cdot \mathrm{L}^{-1}$ de ethephon; $\mathrm{T}_{10} 1500 \mathrm{mg} \cdot \mathrm{L}^{-1}$ de $\mathrm{PBZ}+3 \%$ de nitrato de cálcio; $\mathrm{T}_{11} 1500 \mathrm{mg} . \mathrm{L}^{-1}$ de $\mathrm{PBZ}$ $+3 \%$ de nitrato de cálcio $+1,0$ mL. $L^{-1}$ de ethephon.L; $T_{12} 1500 \mathrm{mg} \cdot \mathrm{L}^{-1}$ de PBZ + 3\% de nitrato de cálcio + 3,0 mL. $\mathrm{L}^{-1}$ de ethephon, com 4 repetições. A mangueira teve um maior florescimento (81,75\%) com $2 \%$ de nitrato de cálcio e $1500 \mathrm{mg}^{-\mathrm{L}^{-1}} \mathrm{de} \mathrm{PBZ}$, o número de frutos por planta teve seu maior valor (86 frutos) com 3\% de nitrato de cálcio e $1500 \mathrm{mg} \mathrm{L}^{-1}$ de PBZ e o peso do fruto foi maior $(425,5 \mathrm{~g}) \mathrm{na}$ dosagem de $3 \mathrm{~mL} . \mathrm{L}^{-1}$ de ethephon. Os produtos aplicados não diferenciaram entre si em relação à produção.
\end{abstract}

Termos para indexação: Mangifera indica L., indução floral, indutores, produção.

\section{FLOWERING AND FRUTIFICATION OF MANGO WITH USE OF PACLOBUTRAZOL, ETHEPHON AND CALCIUM NITRATE}

\begin{abstract}
This work had the objective of testing distinct concentrations of Paclobutrazol, ethephon and calcium nitrate to induce flowering in the production of the mango cv. Tommy Atkins, located in the didactic orchard of the Superior School of Agriculture of Mossoró. The experiment was developped in the year of 1999/2000. A randomized experimental block design, was used in the factorial scheme $2 \times 2 \times 3$; distributed in: $\mathrm{T}_{1} 1000 \mathrm{mg} . \mathrm{L}^{-1}$ of PBZ $+2 \%$ calcium nitrate; $\mathrm{T}_{2} 1000 \mathrm{mg} . \mathrm{L}^{-1}$ of $\mathrm{PBZ}+2 \%$ calcium nitrate $+1,0 \mathrm{~mL} . \mathrm{L}^{-1}$ of ethephon; $\mathrm{T}_{3} 1000 \mathrm{mg} . \mathrm{L}^{-1}$ of PBZ $+2 \%$ calcium nitrate $+3,0 \mathrm{~mL} \cdot \mathrm{L}^{-1}$ of ethephon; $\mathrm{T}_{4} 1000 \mathrm{mg} . \mathrm{L}^{-1}$ of PBZ $+3 \%$ calcium nitrate; $\mathrm{T}_{5} 1000 \mathrm{mg} . \mathrm{L}^{-}$ ${ }^{1}$ of PBZ $+3 \%$ calcium nitrate $+1,0 \mathrm{~mL} \cdot \mathrm{L}^{-1}$ of ethephon; $\mathrm{T}_{6} 1000 \mathrm{mg} \cdot \mathrm{L}^{-1}$ of PBZ $+3 \%$ calcium nitrate $+3,0 \mathrm{~mL} \cdot \mathrm{L}^{-1}$ of ethephon; $\mathrm{T}_{7} 1500 \mathrm{mg} . \mathrm{L}^{-}$ ${ }^{1}$ of PBZ $+2 \%$ calcium nitrate; $\mathrm{T}_{8} 1500 \mathrm{mg} . \mathrm{L}^{-1}$ of PBZ $+2 \%$ calcium nitrate $+1,0 \mathrm{~mL} \cdot \mathrm{L}^{-1}$ of ethephon; $\mathrm{T}_{9} 1500 \mathrm{mg} . \mathrm{L}^{-1}$ of PBZ.L $+2 \%$ calcium nitrate $+3,0 \mathrm{~mL} . \mathrm{L}^{-1}$ of ethephon; $\mathrm{T}_{10} 1500 \mathrm{mg} . \mathrm{L}^{-1}$ of PBZ $+3 \%$ calcium nitrate, $\mathrm{T}_{11} 1500 \mathrm{mg} . \mathrm{L}^{-1}$ of PBZ $+3 \%$ calcium nitrate $+1,0 \mathrm{~mL} . \mathrm{L}^{-1}$ of ethephon; $\mathrm{T}_{12} 1500 \mathrm{mg} . \mathrm{L}^{-1}$ of PBZ $+3 \%$ calcium nitrate $+3,0 \mathrm{~mL} \cdot \mathrm{L}^{-1}$ of ethephon, with 4 replications. The mango had a larger flowering ( $81.75 \%$ of fruits ) with $2 \%$ calcium nitrate and $1500 \mathrm{mg}$. $\mathrm{L}^{-1} \mathrm{of} \mathrm{PBZ}$, the number of fruits per plant was the highest ( 86 fruits) with $3 \%$ calcium nitrate and $1500 \mathrm{mg} \mathrm{L}^{-1}$ of PBZ and the weight of the fruit was the heaviest (425,5g/fruit) in the dosage of $3,0 \mathrm{~mL} . \mathrm{L}^{-1}$ of ethephon. The applied products were not different among themselves in relation to the production.
\end{abstract}

Index terms: Mangifera indica L., floral induction, inductors, production.

\section{INTRODUÇÃO}

A manga é a oitava fruta de importância econômica para o Brasil, tendo uma produção em torno de 23,6 milhões de Mg no ano de 1998, FAO (1999). Esta produção é quase toda consumida no comércio interno, sendo exportado menos de $5 \%$.

O Rio Grande do Norte, juntamente com outros Estados (BA, PE, PI, CE) vêm destacando-se como os principais Estados brasileiros na produção desta fruta, sendo a produção comercializada principalmente no Sudeste, e uma parte exportada para EUA e Europa, tendo como base as cultivares Tommy Atkins, Van Dyke, Haden (Santos et al., 1996)

Os produtores nordestinos vêm enfrentado grandes problemas com a cultura em função de baixos rendimentos alcançados devido a problemas no florescimento e frutificação da planta, sendo que menos de $1 \%$ dos frutos chegam à fase de colheita (Simão, 1971). Com isto, alguns produtos com finalidade de aumentar o rendimento da planta, como nitrato de cálcio, nitrato de potássio, nitrato de amônio, etileno, e outros com a finalidade de restringir o crescimento vegetativo, como

1 Trabalho $n^{\circ}$ 096/2000. Recebido: 09/06/2000. Aceito para publicação: 17/04/2001.

2 Eng. Agrônomo M.Sc. Doutorando em Agronomia-Fitotecnia-UFLA-Lavras, Cx. Postal 37 Lavras-MG, CEP 37.200-000. E-mail vanderm2000@hotmail.com

3 Eng. Agrônomo, Estudante de Pós-Graduação da ESAM, Mossoró-RN, CEP 59625-900

4 Eng. Agrônomo, Prof. Dr. Departamento de Química da ESAM, Mossoró-RN, CEP 59625-900

5 Eng. Agrônomo, Prof. Dr. Departamento de Agricultura da UFLA, Lavras-MG, CEP 37200-000 
Paclobutrazol e Cycocel, estão sendo muito utilizados (Albuquerque, 1992 ; Winston, 1992; Kurian et al., 1993; Ferrari et al., 1996; Salazar-Garcia et al., 1997).Tais produtos, embora indicados para indução do florescimento, necessitam de mais trabalhos que demonstrem suas eficiências no florescimento de mangueira na região Nordeste, uma vez que alguns trabalhos demonstram que estes produtos não tiveram sucesso na indução floral (Pal et al., 1984; Valente \& Donadio, 1993).

A atuação dos produtos à base de nitratos no florescimento ainda é desconhecida. Duas hipóteses são sugeridas por Caldeira (1989): uma afirma que ocorreria produção endógena do etileno a partir da redução do nitrato até a metionina; a outra afirma que altas concentrações de nitrato provocariam estresse físico-químico, estimulando a produção endógena de etileno. $\mathrm{O}$ etileno produzido por estes processos provocaria a indução do florescimento. Contrariamente, Davenport e NunezElisea (1991) não encontraram correlação entre a produção de etileno e a indução floral em mangueira, propondo estes pesquisadores que o etileno não é mediador da iniciação floral. Para Whlley (1993), a ação do nitrato ou paclobutrazol não é indutora, e, sim, meramente desencadeadora ou reforçadora da ação de fatores internos e ambientais na indução floral da mangueira.

O ethephon, descoberto por volta de 1960, quando pulverizado em mangueira em solução aquosa, é prontamente absorvido pelas folhas e translocado na planta. Ao atingir o citoplasma, o ethephon libera o etileno lentamente por meio de reações químicas, permitindo sua ação como indutor floral (Salisbury e Ross, 1991; Taiz e Zeiger, 1991).

Diversos fatores podem influenciar a ação dos indutores, como a temperatura, a concentração de produto na calda, horário, freqüência das aplicações (Winston \& Wright, 1984), falta de adequado estresse hídrico sofrido pelas plantas previamente com relação às aplicações de nitrato (Albuquerque \& Medina, 1991; Tanajura Filho, 1992 e Silva et al., 1994) e o estado nutricional e fisiológico da planta (Castro Neto, 1995).

No Brasil, existe uma grande concentração de produção ao final e início de ano, período em que os preços obtidos não chegam a cobrir os gastos da operação de colheita. Desta forma, o domínio do florescimento torna-se uma prática de grande importância para se obter sucesso com a exportação, como vem ocorrendo nas regiões do Agropolo Mossoró-Açu, no Rio Grande do Norte, e Vale do Rio São Francisco, que estão conseguindo, através da aplicação de indutores, colher frutos mais cedo, no final da entressafra (fevereiro/outubro), uma vez que, nestas regiões, devido às condições climáticas, pode-se produzir manga durante um logo espaço de tempo (setembro/ fevereiro).

Objetivou-se, com este trabalho, verificar o florescimento e a produção da mangueira cv. Tommy Atkins, com o uso do Paclobutrazol-10\% (PBZ), ethephon e nitrato de cálcio na região de Mossoró-RN.

\section{MATERIAL E MÉTODOS}

Este experimento foi instalado e conduzido de maio de 1999 a fevereiro de 2000 no pomar didático da Escola Superior de Agricultura, no município de Mossoró, no Rio Grande do Norte. Foram utilizadas plantas com seis anos de idade da cultivar Tommy
Atkins propagadas pelo processo de enxertia, sobre a cultivar espada como porta-enxerto, plantadas em espaçamento de $8 \mathrm{mx}$ $8 \mathrm{~m}$, e solo tipo arenoso, com sistema de irrigação por microaspersão.

A indução do florescimento iniciou-se no mês de agosto com a primeira aplicação do ethephon, sendo a colheita prevista para o início de dezembro, antecipando, assim, um pouco a colheita, fugindo do período de grande oferta, que normalmente ocorre no final de dezembro e janeiro.

As plantas também foram submetidas a um estresse hídrico durante 20 dias evidenciados por sintomas foliares visuais (curvaturas das margens e aspectos quebradiços).

$\mathrm{O}$ delineamento experimental utilizado foi em blocos casualizados, no esquema fatorial $2 \times 2 \times 3$, assim distribuído: $T_{1}$ $1000 \mathrm{mg} . \mathrm{L}^{-1}$ de PBZ $+2 \%$ de nitrato de cálcio; $\mathrm{T}_{2} 1000 \mathrm{mg} . \mathrm{L}^{-1} \mathrm{de}$ $\mathrm{PBZ}+2 \%$ de nitrato de cálcio $+1,0 \mathrm{~mL} \cdot \mathrm{L}^{-1}$ de ethephon; $\mathrm{T}_{3} 1000$ $\mathrm{mg} . \mathrm{L}^{-1}$ de $\mathrm{PBZ}+2 \%$ de nitrato de cálcio $+3,0 \mathrm{~mL} . \mathrm{L}^{-1}$ de ethephon; $\mathrm{T}_{4} 1000 \mathrm{mg} \cdot \mathrm{L}^{-1}$ de PBZ + 3\% de nitrato de cálcio; $\mathrm{T}_{5} 1000 \mathrm{mg} . \mathrm{L}^{-1} \mathrm{de}$ PBZ + 3\% de nitrato de cálcio $+1,0$ mL. $\mathrm{L}^{-1}$ de ethephon; $\mathrm{T}_{6} 1000$ mg. $\mathrm{L}^{-1}$ de PBZ $+3 \%$ de nitrato de cálcio $+3,0 \mathrm{~mL} . \mathrm{L}^{-1}$ de ethephon; $\mathrm{T}_{7} 1500 \mathrm{mg} . \mathrm{L}^{-1}$ de PBZ $+2 \%$ de nitrato de cálcio; $\mathrm{T}_{8} 1500 \mathrm{mg} . \mathrm{L}^{-1} \mathrm{de}$ $\mathrm{PBZ}+2 \%$ de nitrato de cálcio $+1,0 \mathrm{mLL}^{-1}$ de ethephon; $\mathrm{T}_{9} 1500$ mg. $\mathrm{L}^{-1}$ de PBZ $+2 \%$ de nitrato de cálcio $+3,0 \mathrm{~mL} \cdot \mathrm{L}^{-1}$ de ethephon; $\mathrm{T}_{10} 1500 \mathrm{mg} . \mathrm{L}^{-1}$ de PBZ + 3\% de nitrato de cálcio; $\mathrm{T}_{11} 1500 \mathrm{mg} . \mathrm{L}^{-}$ ${ }^{1}$ de PBZ+ 3\% de nitrato de cálcio $+1,0 \mathrm{mLL}^{-1}$ de ethephon; $\mathrm{T}_{12}$ $1500 \mathrm{mg} . \mathrm{L}^{-1}$ de PBZ + 3\% de nitrato de cálcio + 3,0 mL.L $\mathrm{L}^{-1}$ de ethephon, com quatro repetições.

Para cada parcela, foi utilizada uma planta, e, na análise das variáveis, foram marcados 20 galhos por parcela com média de 85 ramos terminais. Destes galhos, posteriormente, selecionaram-se quatro panículas para análises, escolhidas ao acaso em galhos situados nos quatro quadrantes da planta a uma altura em torno de 2,0 a 3,0 m.

O PBZ foi aplicado via solo, no dia 23-06-99, em covas feitas com as dimensões de $1 \mathrm{~m}$ de comprimento, $20 \mathrm{~cm}$ de largura e $10 \mathrm{~cm}$ de profundidade, a uma distância de $30 \mathrm{~cm}$ do colo da planta. Nesta cova, foi adicionado o produto dissolvido em 2 litros de água nas concentrações conforme os tratamentos. Em torno de sessenta dias após a aplicação do PBZ, foi feita a aplicação do ethephon,via foliar, no dia 25-08-99, com utilização de um pulverizador tratorizado. A aplicação foi feita, dissolvendose o produto nas concentrações, conforme os tratamentos. No dia 09-09-1999, 15 dias após a aplicação do ethephon, iniciaramse as aplicações foliares do nitrato de cálcio, utilizando a mesma metodologia de aplicação usada nas aplicações do ethephon. O nitrato foi aplicado mais quatro vezes, nos dias 16-09-99, 23-0999, 30-09-99 e 06-10-99. Nas aplicações tanto do ethephon como do nitrato, foi utilizado espalhante adesivo (alquil fenol poliglicoléter $250 \mathrm{~g} . \mathrm{L}^{-1}$ ) de nome comercial Adesil, na dosagem de $0,4 \mathrm{~mL} \cdot \mathrm{L}^{-1}$ de calda. As aplicações foram feitas à tarde, por volta de $17 \mathrm{~h}$, sendo utilizados, em média, dez litros da solução por planta, volume suficiente para garantir uma cobertura completa da copa até o ponto do início do escorrimento foliar da calda. Nas aplicações, a umidade relativa e a temperatura média foram de $60 \%$ e $27,5^{\circ} \mathrm{C}$, respectivamente.

As variáveis analisadas: \% de florescimento $(\% \mathrm{~F})$ esta análise foi feita por cinco avaliadores, onde se dava nota de $0 \mathrm{a}$ 10 para o florescimento observado na planta; em seguida, fez-se a média das notas, dando assim a $\%$ de florescimento; tamanho 
da panícula (TP) - obtido através da medida nas panículas previamente marcadas (4); panícula malformada (PMF) - após o completo florescimento, foi observado em cada tratamento se havia presença de panículas malformadas; número de frutos por planta (NFP); peso médio de frutos (PF); produção de frutos por planta (PFP) e por hectare (PFH).

As análises estatísticas foram realizadas através do aplicativo do software SPSSPC, Norusis (1990), e as regressões ajustadas no software Excel. Na análise estatística do NFP, os valores no campo sofreram um ajuste para um mesmo número de ramos terminais (340) com a finalidade de corrigir os eventuais desvios que poderiam ter ocorrido no número desses, na época de amostragem dos galhos nas plantas de cada tratamento (Rabelo, 1995).

\section{RESULTADOS E DISCUSSÃO}

No florescimento, houve interação significativa para os fatores PBZ e nitrato de cálcio. A dose de $1000 \mathrm{mg} . \mathrm{L}^{-1}$ de PBZ não influenciou no florescimento nas duas dosagens de nitrato de cálcio $(\mathrm{P}>0,05)$, porém, quando se aplicou $2 \%$ de nitrato de cálcio, houve maior florescimento (81,75\%) na aplicação de 1500 mg. $\mathrm{L}^{-1}$ de PBZ $(\mathrm{P}<0,05)$, conforme a Tabela 1. Já Pal et al. (1984) e Valente \& Donadio (1993), aplicando diferentes nitratos em concentração de $1 \%$, não encontraram resultado satisfatório na indução floral em mangueira Dashehari e Tommy Atkins.

A aplicação de ethephon neste trabalho não mostrou ser eficiente no aumento do florescimento, resultado este que está de acordo com Valente \& Donadio (1993), que também observaram que a aplicação do ethephon não foi eficiente em promover o florescimento em mangueira.

O tamanho da panícula não apresentou variação significativa, demonstrando que nenhum dos tratamentos teve influência no seu crescimento, embora Kurian et al. (1993), em seu trabalho, observaram uma redução no tamanho das panículas quando utilizaram $1250 \mathrm{mg} \cdot \mathrm{L}^{-1}$ de $\mathrm{PBZ}$ em cultivares de manga Alphonso II.

O número de frutos por planta não foi diferente em nenhum dos tratamentos utilizados isoladamente, porém houve interação entre os três produtos utilizados, demonstrando que há uma relação positiva entre os três produtos em aumentar o número de frutos por planta.
Na Figura 1, observa-se que o maior número de frutos por planta (86 frutos) foi obtido com 3,0 mL.L $\mathrm{L}^{-1}$ de ethephon, $3 \%$ de nitrato de cálcio e $1500 \mathrm{mg} . \mathrm{L}^{-1}$ de PBZ, e o menor (39 frutos), com $1000 \mathrm{mg} . \mathrm{L}^{-1}$ de $\mathrm{PBZ}+2 \%$ de nitrato de cálcio sem a aplicação do ethephon.

Este resultado vem confirmar a resposta positiva do Paclobutrazol no rendimento da mangueira, confirmado também por Kurian et al. (1993), Ferrari et al. (1996) e Albuquerque (1992), que encontraram respostas positivas no aumento do número de frutos por planta ao analisar trabalhos feitos em mangueira de diferentes cultivares, com a aplicação do Paclobutrazol, e também confirmam os encontrados por Santana et al. (1997), que, aplicando nitrato de cálcio a 4\% em variedades Haden e Tommy Atkins, promoveram um aumento significativo no florescimento e no número de frutos por planta.

$\mathrm{O}$ peso do fruto só foi influenciado no tratamento com ethephon. Na Figura 2, observa-se que, à medida que se aumenta a dosagem de ethephon há também aumento no peso do fruto, sendo que, na dosagem de $3,0 \mathrm{~mL} . \mathrm{L}^{-1}$ de ethephon, o peso do fruto ficou em $425,5 \mathrm{~g}$, cerca de $16,7 \%$ a mais em relação ao tratamento sem a aplicação deste produto, embora Ferrari et al. (1996) tenham encontrado resposta significativa em relação ao peso total dos frutos quando utilizaram Paclobutrazol nas dosagens de $500 \mathrm{mg} . \mathrm{L}^{-1}$ e $750 \mathrm{mg} . \mathrm{L}^{-1}$; neste trabalho, esta variável não foi influenciada pelo Paclobutrazol, concordando com Winston (1992), que também não encontrou resposta significativa em relação ao peso médio de frutos de manga cv. Kensigton Pride na utilização de Paclobutrazol.

A produção de frutos por planta e por hectares não apresentou significância até 5\% pelo teste $\mathrm{F}$, em nenhum dos tratamentos aplicados, embora o tratamento com a utilização de $1500 \mathrm{mg} . \mathrm{L}^{-1}$ de PBZ, 3, 0 mL.L $\mathrm{L}^{-1}$ de ethephon e 3\% de nitrato de cálcio apresentasse a maior média de produção $(38,34 \mathrm{Kg} /$ planta), cerca de $155,8 \% \%$ a mais em relação ao tratamento com 1500 mg. $\mathrm{L}^{-1}$ de PBZ, 2,0 mL.L ${ }^{-1}$ de ethephon e $2 \%$ de nitrato de cálcio e que ficou em torno de 14,98kg/planta. Estas respostas indicam uma tendência no aumento da produção com o aumento da dosagem de ethephon e de nitrato de cálcio. Estas respostas discordam de vários trabalhos com a utilização destes produtos na indução floral em mangueira onde, na maioria, há aumento bastante significativo na produção (Winston, 1992; Kurian et al., 1993, Ferrari et al.,1996; Albuquerque et al., 1992, e Santana etal., 1997).

TABELA 1 - Análise de variância do desdobramento de interação $\mathrm{Ca}\left(\mathrm{NO}_{3}\right)_{2}$ e PBZ da variável \% de Florescimento. Mossoró/RN, fevereiro de 2000.

\begin{tabular}{|c|c|c|}
\hline & $20 \mathrm{ml} / \mathrm{planta} \mathrm{PBZ}$ & $30 \mathrm{ml} / \mathrm{planta} \mathrm{PBZ}$ \\
\hline $2 \%$ de $\mathrm{Ca}\left(\mathrm{NO}_{3}\right)_{2}$ & $63,00 \mathbf{A}^{*}$ & $81,75 \mathbf{B}$ \\
\hline $3 \%$ de $\mathrm{Ca}\left(\mathrm{NO}_{3}\right)_{2}$ & $76,50 \mathbf{A}$ & $69,50 \mathbf{A}$ \\
\hline $\mathrm{CV}$ & 22,54 & 27,90 \\
\hline
\end{tabular}

Médias seguidas de mesma letra nas linhas não diferem entre si pelo teste $\mathrm{F}(\mathrm{P}>0,05)$. 


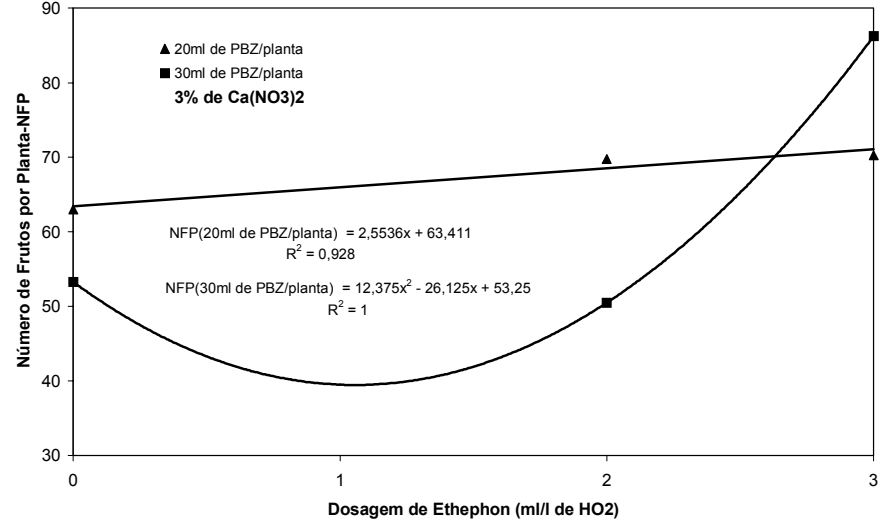

FIGURA 1 - Equação da interação Paclobutrazol x nitrato de cálcio $\mathrm{x}$ Ethephon para a variável número de frutos por planta. Mossoró/RN, fevereiro de 2000.

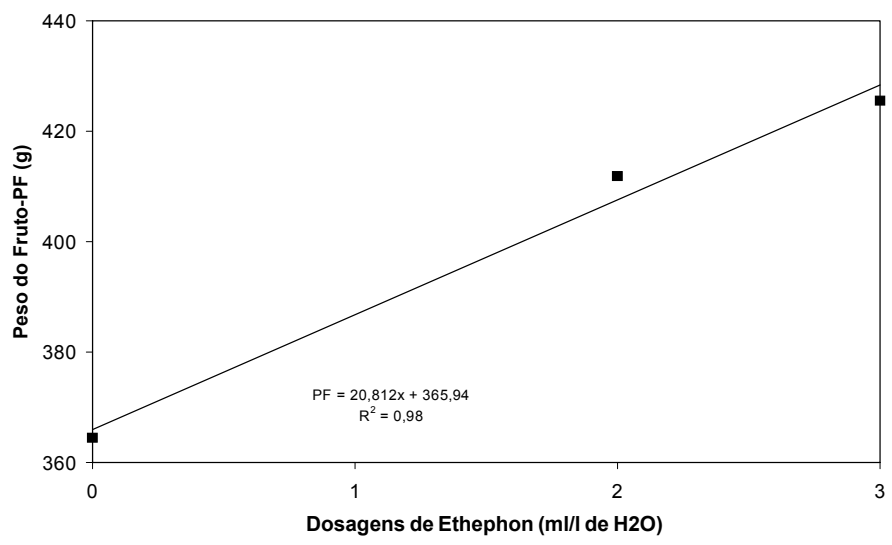

FIGURA 2 - Equação de regressão do tratamento com ethephon para a variável peso dos frutos. Mossoró/RN, fevereiro de 2000.

\section{CONCLUSÕES}

Nas condições em que foi desenvolvido este trabalho, chegouse às seguintes conclusões:

1 - O florescimento apresentou-se melhor com $2 \%$ de nitrato de cálcio e $1500 \mathrm{mg} . \mathrm{L}^{-1}$ de PBZ.

2 - O maior número de frutos por planta foi alcançado com $3 \%$ de nitrato de cálcio e $1500 \mathrm{mg} . \mathrm{L}^{-1}$ de PBZ.

3 - A dosagem de $3,0 \mathrm{~mL} \cdot \mathrm{L}^{-1}$ de ethephon proporcionou maiores pesos nos frutos.

4 - Os produtos aplicados não se diferenciaram entre si em relação à produção.

\section{AGRADECIMENTOS}

Agradecemos ao Prof. Dr. José Celesmário Tavares/ ESAM-Mossoró-RN, pela ajuda durante este trabalho e ao prefeito do Câmpus, Wilson Galdino, pela doação da área experimental.

\section{REFERÊNCIAS BIBLIOGRÁFICAS}

ALBUQUERQUE, J.A S. ; MEDINA, V. A. D. Indução de floração em mangueira cv. Tommy Atkins com nitrato de amônio. Revista Brasileira de Fruticultura, v. 13, p. 5-93, 1991.

ALBUQUERQUE, J.A.S. Uso de produtos químicos e práticas culturais para indução da floração da mangueira na região do submédio São Francisco. Revista Brasileira de Fruticultura,v. 14, p. 82-177, 1992.

ALBUQUERQUE, L.A.S.; MOUCO, M.A.; REIS, V.C. Floração da mangueira através do uso de reguladores de crescimento. Petrolina: EMBRAPA, 1999. v. 12 (Instrução Técnicas).

CALDEIRA, M.L. Indução química do florescimento em manga. In: SIMPÓSIO SOBRE MANGICULTURA, 2., Jaboticabal, 1989. Anais... Jaboticabal. FUNEP, 1989. p.63-157.

CASTRO NETO, M.T. Aspectos fisiológicos da mangueira sob condições irrigadas. EMBRAPA. In: Informações técnicas sobre a cultura da manga no semi-árido brasileiro. 1995. 173p.

DAVENPORT, T.L.; NUÑES-ELISEA, R. Is endogenous ethylene involved in mango floral induction. Third International mango Symposium. Acta Horticulturae, Wageningen, v. 291, p.85-94, 1991.

FAO (1999) http://apps.fao.org/lim500/nph weap.pl

FERRARI, F.D.; SERGENT, E.A. Promocion de la floración y frutificacion del mango ( Mangifera indica L.) cv. Haden, com Nitrato de Potasio. Revista Faculdade Agronomia, Maracay, v. 22, p.1-8, 1996.

FERRARI, F.D.; SERGENT, E.A. Promocion de la floración y frutificacion del mango ( Mangifera indica L.) cv. Haden, com Paclobutrazol. Revista Faculdade Agronomia, Maracay, v. 22, p. 9-17, 1996.

KURIAN, R.M.; IYER, C.P.A.Chemical regulation of tree size in mango (Mangira indica L.) cv. Alphonso.I.Effects of growth retardant treatments on vegetative growth and tree vigour. Journal of Horticultural Science, Ashford, v.68, p. 349-354, 1993

NORUSIS, M. J. SPSS statistics. IIIinois: SPSS. Inc, 1990.

PAL, R.N.; CHADKA, K.L.; RAO, M.R.K Effect of different plant growth regulators and other chemicals on flowering behaviour of mango. Indian Journal of Horticultural Science, Ashford, v. 41, p. 8-15, 1984.

RABÊLO, J.E. de S. Florescimento e frutificação de mangueira (Mangifera indica $L$.) 'Haden' em resposta a anelamento e aplicação de ethephon e nitrato de potássio. 1995. 84p. Dissertação (Mestrado em Agronomia) - Universidade Federal de Viçosa, Viçosa, 1995.

SALAZAR-GARCIA, S.; VAZQUEZ-VALDINA, V. Physiological persistence of Paclobutrazol on the Tommy Atkins mango 
(Mangifera indica L.) under rainfed conditions. Journal of Horticultural Science, Ashford, v. 72, n. 2, p. 339-345, 1997.

SALISBURY, F.B. ; ROSS, C.W. Plant physiology. Belmont: Wadsworth, 1991.682p.

SANTANA, J.R.F.de; CUNHA, G.A.P. da; FONSECA, N.; SOUTO, R.F. Efeito de indutores florais sobre o florescimento, frutificação e rendimento das cvs. de manga Van Dyke, Haden e Tommy Atkins no Norte de Minas Gerais. 1 - Nitrato de Cálcio. Revista Brasileira de Fruticultura, Cruz das Almas, v.19, n.2.p.159-167, dez. 1997.

SANTOS, M.G.F.M.; ALVES, M.C.S.; TAVARES, J.C. Perfil da exportação da mangueira no Estado do Rio Grande do Norte. In: SÃO JOSÉ, A. R.; SOUZA, I.V.B.; MARTINS FILHO. J.; MORAIS, O.M. Manga: tecnologia de produção e mercado. Vitória da Conquista-BA.1996.p 338-346.

SILVA, D. A. M.; VIEIRA, V.J.S.; MELO, J.J.L.; ROSA JUNIOR, C.R.M.; SILVA FILHO, A.V. Mangueira (Mangifera indica L.): cultivo sob condição irrigada. Recife: SEBRAE, 1994. 42p. ( Agricultura, 9).

SIMÃO, S. Manual de fruticultura. São Paulo: Ceres, 1971. 530p.

TAIZ, L.; ZEIGER, E. Plant physiology. Redwood: Benjamin/ Cummings, 1991.565p.
TANAJURA FILHO, J.G. Indução de florescimento em mangueira In:SÃO JOSÉ, A.R. SOUZA, I.V.B. In:SIMPÓSIO ESTADUAL SOBRE PRODUÇÃO DE MANGA, 1., 1990, Vitória da Conquista, 1990. Anais... Vitória da Conquista, UESB, 1992. P.5-83.

VALENTE, J.P.; DONADIO, L.C. Mango flower induction by $\mathrm{KNO}_{3}$ and ethephon spraying in:INTERNATIONAL SYMPOSIUM ON TROPICAL FRUITS, 1., 1993, Vitória. Abstracts... Vitória: EMCAPA, 1993. p.51.

WAMPLE, R.L; E.B.CULVER The influence of Paclobutrazol, a new growth regulator, on sunflowers. Journal of the American Society for Horticulture Science, Alexandria, v.108, p.122-125, 1983.

WHILEY, A. Environmental effects on phenology and physiology of mango. A review. Fourth International mango Symposium. Miami Beach, Florida. Acta Horticulturae. Wageningen, v. 341, p. 168-176, 1993.

WINSTON, E.C. Evaluation of Paclobutrazol on growth, flowering and yield of mango c.v. Kensington Pride. Australian Journal of Experimental Agriculture, Collingwood, v. 32, p.97-104, 1992.

WINSTON, E.C. ; WRIGHT, R.M. Mango flower indiction:ethephon, potassium nitrate and cincturing In:AUSTRALIAN MANGO RESEARCH WORKSHOP, 1., Cairns. Proceedings... Cairns. CSIRO, 1984. p.57-70. 